\title{
Grado de satisfacción del usuario de ciclismo indoor en los centros deportivos de Granada

\author{
Indoor cycling client's satisfaction level in sports clubs in Granada
}

\author{
Jaime Morente Sánchez, Pedro Yaques Arioni, Mikel Zabala Díaz \\ Universidad de Granada
}

\begin{abstract}
Resumen .El objetivo del presente estudio fue conocer y comparar el grado de fidelidad y de satisfacción del practicante de ciclismo indoor en Granada a nivel general y atendiendo al género y al tamaño su centro deportivo (grande vs pequeño). Un total de 148 sujetos (31.8 \pm 9.9 años; 62 hombres) procedentes de 12 centros deportivos (75 de centros grandes; 73 de centros pequeños) conformaron la muestra. Este estudio piloto se presenta bajo un diseño descriptivo de tipo transversal a través de un cuestionario validado. Se observó que el usuario, en mayor medida de género femenino, de esta actividad es fiel y no esporádico. Ellos están más satisfechos que ellas, mientras que el trato ofrecido por el monitor se presenta como una de las acciones más valoradas de cara a la satisfacción del cliente. El grado de satisfacción y de fidelización del cliente de ciclismo indoor del área metropolitana de Granada es notable. En líneas generales, no se encontraron diferencias importantes entre centros grandes y pequeños, ni entre hombres y mujeres.

Palabras clave. spininng, cliente, eficacia, actividad, fitness.
\end{abstract}

Abstract. The aim of this study was to know and compare indoor cycling client' satisfaction and commitment levels in sports clubs and fitness centers in Granada, in general, and taking into account the sport center size (large vs small) and gender. Total sample was composed of 148 subjects (31.8 \pm 9.9 years; 62 men) from 12 sports centers (75 from large size sport center, 73 from small sport centers). This descriptive cross-sectional pilot study was carried out by means of a validated questionnaire. Indoor cycling users s are mostly female and non-sporadic. Males were more satisfied than females, while elderly participation is very low. The way participants were treated by the monitor was considered as one of the most valued and most important factors in relation with customers satisfaction level. Significant differences were not found betwen the so-called large and small fitness centers. The indoor cycling users' level of satisfaction and commitment in the metropolitan area of Granada was remarkable. Overall, no significant differences between so-called large centers and small centers were found. Women, despite being less satisfied than men, attend more regularly.

Keywords: spinning, customer, efficacy, activity, fitness.

\section{Introducción}

En los últimos 15 años se ha producido un cambio en la tendencia de práctica de actividades físicas de la población en España como nos señalan los estudios, de García Ferrando (2001, 2006, 2010) sobre los hábitos de práctica deportiva. En este tiempo se ha producido un incremento considerable de nuevas actividades y de sus requeridas instalaciones deportivas dirigidas a todos los públicos García Ferrando (2006) y Ferrando y Llopis Goig (2011).

Se ha observado un cierto cambio en términos de práctica deportiva en la población española aumentando un tipo de práctica físico-deportiva orientada hacia la salud dentro de una corriente corporal narcisista (Otero, 2004). En la última «Encuesta de Hábitos Deportivos de los españoles» García Ferrando y Llopis Goig (2011) en relación a la inmediatamente anterior (García Ferrando, 2006), se pueden observar notorias diferencias. Atendiendo a los datos de 2006, el 70\% de las personas que practican deporte lo hacían sin preocuparse de competir, figurando entre los motivos de práctica más destacados el realizar ejercicio físico (60\%), la diversión (47\%), el mero gusto por el deporte (34\%) así como mantener y/o mejorar la salud(16\%). En laúltima versión de esta misma encuesta llevada a cabo en 2010 García Ferrando y Llopis Goig (2011), sólo el 5\% solicitaba la actividad física para canalizar su ganas de competir, mientras que la inmensa mayoría declaraba otras motivaciones tales como hacer ejercicio físico (70\%), diversión (50\%), porque le gusta el deporte como tal (41\%) y/o por mantener y/o mejorar la salud (48\%). En nuestro país, este interés por el fomento de la actividad física, sobre todo en términos recreativos y de salud como se observa a partir de la última encuesta, se ha concretado en diversos marcos legales tanto en el ámbito educativo como en el de la gestión pública del deporte.

Dentro de esta corriente de cambio una de las actividades que encaja perfectamente en el perfil de la nueva tendencia es el ciclismo indoor o Spinning (término incorrecto pues hace uso de una marca comercial registrada) (Sanz, Redondo, Gutiérrez y Cuadrado, 2005). Esta es una actividad grupal dirigida en la cual se hace uso de bicicletas estáticas

Fecha recepción: 25-03-14- Fecha envío revisores: 26-03-14- Fecha de aceptación: 11-06-14 Jaime Morente Sánchez jaimemorente@ugr.es diseñadas especialmente por y para esta actividad y que cuenta con un ajuste de carga regulable. Anivel condicional o físico, la principal finalidad de la actividad es cardiovascular, pero como es lógico el trabajo de fuerza a nivel del tren inferior es considerable. El ciclismo indoor lleva más de diez años siendo una de las actividades de más éxito en los centros deportivos. Su auge está motivado principalmente por el mínimo aprendizaje que requiere, la facilidad de ejecución, la diversión y el tiempo necesario para conseguir los objetivos que se persiguen. La actividad está presente en todos los centrosde fitness hoy en día y supone una herramienta fidelizadora de clientes muy poderosa (LunaArocas, Mundina y Gómez, 1998). Es una actividad utilizada como vehículo para captar y mantener clientes a la que los clientes pues los fideliza con gran facilidad. Hoy día, se construyen crean centros dedicados casi exclusivamente a esta actividad siendo complementados con muy pocos servicios más (Luna y Tang, 2005).

Tras una revisión de la literatura científica y divulgativa al respecto hemos detectado cierta falta de estudios en relación al grado de satisfacción percibido por el cliente de ciclismo indoor (Luna-Arocas y Mundina, 1998; Papadimitriou y Karteroliotis, 2000; Lofshult, 2003; Bodet, 2006). No obstante, reconocemos la existencia de un artículo semejante (Sanz et al., 2005) desarrollado en otra parte de España y hace cerca de una década. El uso de la misma herramienta, nos permitirá establecer comparaciones entre resultados así como extraer conclusiones específicas a este tipo de práctica.

Para desarrollar el presente estudio piloto se han considerado una serie de parámetros a partir de los cueles se podrás predecir si, en el futuro, el cliente continuará desarrollando la misma actividad lo cual nos dará información de cara a una subsecuente toma decisional tal como reforzar o mantener algún aspecto de este tipo de práctica. Esta evaluación debería ser objeto de preocupación para los gerentes de los centros deportivos y salas de fitness ya que es sinónimo de lo que sucede en la realidad diaria del centro y permitirá mantener, evitar la pérdida e incluso captar clientes para dicha actividad y, muy probablemente teniendo simultáneamente la oportunidad de vender otros servicios (Shonk y Chelladurai, 2008; Westerbeek y Shilbury, 2003). «La calidad del servicio es la clave de la satisfacción del cliente, para ello es necesario saber qué quiere tu cliente o usuario» (Martínez-Tur, Peiró y Ramos, 2001). 
Consideramos que debido a la escasez de este tipo de trabajos a nivel nacional centrados en esta modalidad de actividad físico-deportiva tan de moda en la actualidad podría resultar interesante describir de forma general quétipo de público es el que realiza ciclo indoor, con qué frecuencia, cuánto tiempo lleva como usuario, si disfruta o no de la actividad, si alcanza sus objetivos y cuáles son los aspectos positivos y negativos en relación a su práctica como tal. Del mismo modo, resulta de gran interés comparar el grado de satisfacción y fidelidad entre los usuarios en función del género como tal y atendiendo al tipo de centro en el que realizan la actividad, respectivamente. Por todo ello, el objetivo del presente estudio fue conocer y comparar el grado de fidelidad y de satisfacción del practicante de ciclismo indoor en Granada a nivel general general y atendiendo al género y al tamaño su centro deportivo (grande vs pequeño).

\section{Material y Métodos}

\section{Participantes}

Un total de 148 sujetos (31.8 \pm 9.9 años) de 12 centros deportivos, 75 procedentes de centros grandes y 73 de centros pequeños, conformaron la muestra (tabla 1). El 58.1\% (86) son mujeres mientras que el resto 41.9\% (62) son hombres. El practicante más joven tenía 16 años y el mayor 60.

Tabla 1.

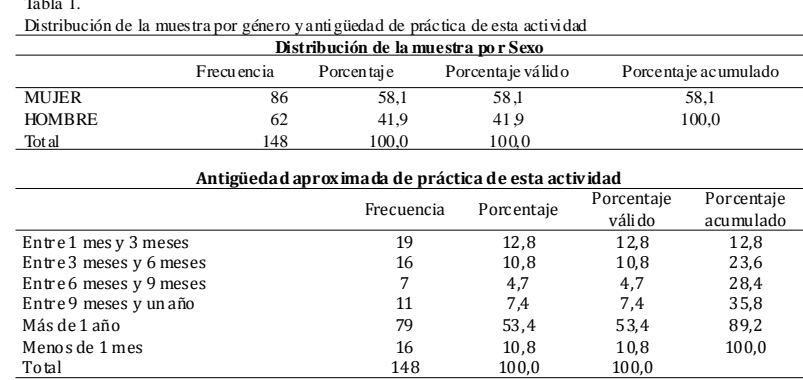

\section{Diseño}

Se estableció un diseño descriptivo de tipo transversal. La muestra obtenida aleatoriamente se obtuvo asumiendo un nivel de confianza del $95 \%$ y un intervalo de confianza del $10 \%$.

En nuestro caso, para este estudio piloto, hemos seleccionado el área metropolitana de Granada como población de estudio. Se han seleccionado los centros aleatoriamente y se han clasificado de manera simple, teniendo en cuenta previamente el número de centros grandes y centros pequeños. Los centros grandes, «macrocentros de Fitness», son los centros de nueva generación, preparados y construidos para recibir a una gran cantidad de usuarios. Los centros pequeños son aquellos, normalmente más antiguos, con una infraestructura más pequeña y con un volumen de socios claramente inferior.

Los centros grandes fueron We, O2, Yo10, y Spazio Reebook (la media de participantes fue de 30 participantes en 4 centros con 12 grupos que repiten sesión en la semana, conformando una $\mathrm{N}$ potencial total aproximada de 1440 participantes), y los considerados pequeños fueron Paladium, TNT, Universal Fitness, Triunfo, Nueva Era, San Juan de Dios, Elite Fitness, Alpha Gim y Palestra (la media de participantes se promedió en 12 para los 8 centros con 6 grupos que repiten sesión en la semana, conformando una $\mathrm{N}$ potencial total aproximada de 576 participantes).

\section{Instrumentos}

Se procedió a la obtención de datos a través de una versión online de un cuestionario validado para evaluar la satisfacción de los clientes de ciclo indoor (Sanz et al., 2005). Esta herramienta esta compuesta por una escala Likert de 1 (muy en desacuerdo) a 5 (muy de acuerdo) sobre la cual los clientes deben posicionarse en relación a una serie de ítems relacionados con ciertos aspectos. Del cuestionario de satisfacción se pueden extraer cinco categorías de variables que configuran la satisfac- ción del cliente en diferentes áreas: las relacionadas con el monitor (ítems $5,13,19,22$ y 26), con la sala donde se realiza la actividad (ítems 7, 9, 10, 14, 15, 18 y 27), con las bicicletas (ítems 4, 8, 24 y 29), con la organización general de la actividad (ítems 2, 3, 6, 16 y 20) y otros aspectos (ítems 1, 11, 12, 23 y 30).

Se recurrió a la tecnología Google Drive, concretamente a la herramienta de creación y gestión de cuestionarios, para almacenar los resultados en una hoja de cálculo Excel. Para el tratamiento de los datos utilizamos el paquete estadístico SPSS IBM 20.0.

\section{Procedimiento}

En primer lugar, se llevó a cabo un protocolo de captación de personal participante, y para ello se procedió a la búsqueda de los gimnasios, centros deportivos y clubs de fitness del área metropolitana de Granada. Se diseñaron e hicieron llegar una serie de solicitudes formales de colaboración e información sobre el objetivo del estudio a todos los centros indexados en un documento, «Páginas amarillas», de carácter público y fácil acceso. Una vez conseguida la aceptación por parte de los gerentes y directores técnicos de los respectivos centros, accedimos directamente a ellos. Previamente al inicio de las sesiones se explicaba a los clientes en qué consistía exactamente el estudio y se les solicitaba su participación a posteriori. Por lo tanto, con el fin de no romper la dinámica de las sesiones y para que los clientes pudieran contestar de forma voluntaria y anónima, se les solicitó su correo electrónico. A continuación, procedimos a enviar la versión digital del cuestionario a la que accedía mediante un link incorporado al propio cuerpo del email. Los clientes disponían de una semana de plazo para completar el cuestionario desde casa.

\section{Análisis estadístico}

Para el tratamiento de los datos se recurrió al software Statistical Package for the Social Sciences (IBM-SPSS 20.0). Tras realizar la prueba denormalidad(Kolmogorov-Smirnov) y confirmar la distribución no normal de las variables (característica propia en este tipo de escalas Likert) se procedió a la realización de análisis de correlación de carácter bivariado de Spearman y una prueba no paramétrica de contraste para muestras independientes (test de Mann Whitney), comparando los centros pequeños respecto a las grandes superficies y mujeres respecto a hombres. Se tomó como referencia un valor de $\mathrm{p}<.05$ para determinar diferencias significativas.

\section{Resultados}

De acuerdo con Ashby (2000), se enuncian los datos referenciando a las tablas contenedoras de los mismos para facilitar el entendimiento Tabla 2.

\begin{tabular}{|c|c|c|c|c|c|}
\hline & \multirow[b]{2}{*}{ ÍTEM } & \multicolumn{2}{|c|}{$\begin{array}{c}2005 \\
\text { Norte España } \\
\end{array}$} & \multicolumn{2}{|c|}{2011 Granada } \\
\hline & & & & Media & SD \\
\hline 1 & Con esta actividad ob tengo los resultados que esperaba. & 4,19 & 0,85 & 4,29 & 0,75 \\
\hline 2 & Estoy in formado/a sobre los beneficios de esta activi dad. & 3,81 & 1,27 & 3,89 & 1,01 \\
\hline 3 & Me gusta el horario en que se desarrol la la actividad. & 4,09 & 1,16 & 4,35 & 0,86 \\
\hline 4 & Las bicicletas están bien cuidadas. & 3,77 & 1,17 & 3,80 & 1,07 \\
\hline 5 & El profesor es respetuoso con el horario. & 4,82 & 0,54 & 4,62 & 0,65 \\
\hline 6 & Estoy satisfecho/a con la relación calidad-precio de la actividad. & 4,36 & 0,88 & 4,09 & 0,89 \\
\hline 7 & La sala tiene una temperatu ra adecu ada. & 3,81 & 1,05 & 3,51 & 1,18 \\
\hline 8 & Las bicicletas funcionan bien. & 3,64 & 1,15 & 3,79 & 1,04 \\
\hline 9 & La sala está bien venti lada durante la acti vidad. & 3,42 & 1,25 & 3,28 & 1,12 \\
\hline 10 & Considero que el vol umen de la mús ica es idóneo. & 4,32 & 0,86 & 4,07 & 0,82 \\
\hline 11 & Las sesiones me resultan monótonas. & 4,20 & 1,14 & 2,39 & 1,25 \\
\hline & La inten sidad de las sesiones es la & 4,45 & 0,67 & 4,17 & 0,79 \\
\hline 13 & Estoy contento/a con el trato recibid op or el profesor. & 4,82 & 0,52 & 4,45 & 0,74 \\
\hline 14 & Me gusta la distribución que tienen las bicicletas en la sala. & 4,28 & 0,91 & 3,71 & 1,13 \\
\hline & La sala me resulta acogedora. & 4,04 & 0,95 & 3,60 & 1,14 \\
\hline & Existen vari os aspectos mejorables en es & & & 3,43 & 1,02 \\
\hline 17 & La duración de las clases me parece adecuada. & 4,51 & 0,72 & 4,34 & 0,72 \\
\hline 18 & El tipo de música me resul ta apropiado. & 4,58 & 0,67 & 4,11 & 0,83 \\
\hline 19 & Considero que el grado de implic ación del mon itor es bueno. & 4,72 & 0,57 & 4,46 & 0,73 \\
\hline 20 & $\begin{array}{l}\text { Estoy contento/a con el número de h cas semanales dedicadas a esta } \\
\text { actividad. }\end{array}$ & 4,08 & 1,20 & 4,41 & 0,77 \\
\hline 21 & Practicar esta act ividad me ayuda a rel aj arme. & 4,53 & 0,73 & 4,41 & 0,86 \\
\hline 22 & $\begin{array}{l}\text { Al desarrollarla práctica, el monitor ti ene en cuenta las di ferencias } \\
\text { individuales. }\end{array}$ & 3,97 & 1,20 & 3,60 & 1,13 \\
\hline 23 & Me divierto mucho practic ando esta actividad. & 4,59 & 0,62 & 4,32 & 0,87 \\
\hline & Las bicicletas me result an cómodas. & 3,72 & 1,04 & 3,72 & 1,04 \\
\hline 25 & La relación con los compañeros es agradable. & 4,32 & 0,87 & 4,18 & 0,86 \\
\hline 26 & Considero que el monitor anima adecuadamen te al grupo. & 4,67 & 0,65 & 4,39 & 0,78 \\
\hline 27 & La sala siempre está limpia antes de su uti lización. & 4,42 & 0,96 & 4,24 & 1,03 \\
\hline 28 & Alguna vez he pensado abandonar la actividad. & & & 1,86 & 1,12 \\
\hline 29 & Las bicicletas siempre están limpias antes de su uti lización. & 4,18 & 1,05 & 3,95 & 1,20 \\
\hline 30 & A veces termino con dolor de cabeza. & 4,46 & 1,16 & 1,50 & 0,82 \\
\hline
\end{tabular}




\begin{tabular}{|c|c|c|c|c|c|c|c|c|c|c|c|c|}
\hline & \multicolumn{2}{|c|}{ Todos $\mathrm{n}=148$} & \multicolumn{2}{|c|}{ Centros grandes $\mathrm{n}=75$} & \multicolumn{2}{|c|}{$\begin{array}{c}\text { Centros pequeños } \\
\mathrm{n}=73\end{array}$} & \multirow{2}{*}{$\mathrm{P}$} & \multicolumn{2}{|c|}{ Hombres $\mathrm{n}=62$} & \multicolumn{2}{|c|}{ Mujeres $\mathrm{n}=86$} & \multirow{2}{*}{$\mathrm{P}$} \\
\hline & $\mathrm{M}$ & SD & M & SD & M & SD & & M & SD & M & SD & \\
\hline \multicolumn{13}{|c|}{ Satisfacción con el monitor } \\
\hline El profesor es respet uoso con el horario. & 4,62 & 0,65 & 4,6 & 0,59 & 4,64 & 0,71 & NS & 4,63 & 0,75 & 4,62 & 0,58 & NS \\
\hline Estoy conten to/a con el trato recibido por el profesor. & 4,45 & 0,74 & 4,44 & 0,76 & 4,45 & 0,72 & ,032 & 4,61 & 0,64 & 4,33 & 0,79 & 016 \\
\hline Considero que el grado de implic ación del monitor es bueno. & 4,46 & 0,73 & 4,47 & 0,77 & 4,45 & 0,7 & NS & 4,5 & 0,76 & 4,43 & 0,71 & NS \\
\hline $\begin{array}{l}\text { Al desarrollar la práctica, el monitor tiene en au enta las } \\
\text { di ferencias individu ales. }\end{array}$ & 3,6 & 1,13 & 3,6 & 1,13 & 3,6 & 1,14 & NS & 4,65 & 1,01 & 3,57 & 1,21 & NS \\
\hline Consid ero que el monitor ani ma adecuadam ente al grupo. & 4,39 & 0,78 & 4,41 & 0,78 & 4,36 & 0,78 & NS & 4,48 & 0,76 & 4,31 & 0,79 & NS \\
\hline \multicolumn{13}{|c|}{ Satisfacción con la sala } \\
\hline La sal a ti ene una tem perat ura adecuada. & 3,51 & 1,18 & 3,71 & 1,12 & 3,32 & 1,21 & NS & 3,6 & 1,15 & 3,45 & 1,2 & NS \\
\hline La sal a está bien vent ilada durante la actividad. & 3,28 & 1,12 & 3,53 & 1,01 & 3,03 & 1,17 & NS & 3,39 & 1,08 & 3,2 & 1,16 & NS \\
\hline Considero que el volumen de la música es idóneo. & 4,07 & 0,82 & 4,08 & 0,88 & 4,05 & 0,77 & NS & 3,98 & 0,88 & 4,13 & 0,78 & NS \\
\hline Me gusta la distribución que tienen las bicicetas en la sala. & 3,71 & 1,13 & 3,86 & 1,03 & 3,56 & 1,2 & NS & 3,66 & 1,1 & 3,74 & 1,15 & NS \\
\hline La sala me resulta acogedora. & 3,6 & 1,14 & 3,86 & 0,99 & 3,35 & 1,22 & NS & 3,55 & 1,17 & 3,64 & 1,13 & NS \\
\hline El tipo de mú sica me resulta apropiado. & 4,11 & 0,83 & 4,01 & 0,84 & 4,2 & 0,82 & NS & 4,06 & 0,87 & 4,14 & 0,81 & NS \\
\hline La sala siempre está limpia an tes de su uti lización. & 4,24 & 1,03 & 4,42 & 0,91 & 4,07 & 1,11 & NS & 4,39 & 0,82 & 4,14 & 1,15 & NS \\
\hline \multicolumn{13}{|c|}{ Sa tisfacción con las bicicletas } \\
\hline Las biciclet as están bien auidadas. & 3,8 & 1,07 & 4,03 & 0,94 & 3,59 & 1,14 & NS & 3,77 & 1,11 & 3,83 & 1,04 & NS \\
\hline Las bicicletas fun cionan bien. & 3,79 & 1,04 & 3,99 & 0,91 & 3,6 & 1,14 & NS & 3,82 & 1,08 & 3,77 & 1,03 & NS \\
\hline Las bicicletas me resultan cómodas. & 3,72 & 1,04 & 3,90 & 1,04 & 3,55 & 1 & ,003 & 4,03 & 0,9 & 3,5 & 1,07 & ,003 \\
\hline Las bicid etas siempre están limpias antes de su uti lización. & 3,95 & 1,2 & 4,19 & 1,06 & 3,71 & 1,28 & NS & 4 & 1,17 & 3,91 & 1,22 & NS \\
\hline
\end{tabular}

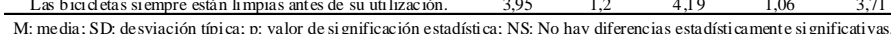

Tabla 4.

$\underline{\text { Comparativa ítems relacionados con grado de satisfacción con la organización, con otros aspectos ygeneral. }}$

\begin{tabular}{|c|c|c|c|c|c|c|c|c|c|c|c|c|}
\hline & \multicolumn{2}{|c|}{ Todos $n=148$} & \multicolumn{2}{|c|}{$\begin{array}{c}\text { Centros grandes } \\
n=75\end{array}$} & \multicolumn{2}{|c|}{$\begin{array}{c}\text { Centros pequẽos } \\
n=73\end{array}$} & \multirow[t]{2}{*}{$\mathrm{P}$} & \multicolumn{2}{|c|}{ Hombres $n=62$} & \multicolumn{2}{|c|}{ Mujeres $n=86$} & \multirow[t]{2}{*}{$\mathrm{P}$} \\
\hline & $\mathrm{M}$ & SD & & & $\mathrm{M}$ & SD & & $\mathrm{M}$ & $\mathrm{SD}$ & $\mathrm{M}$ & SD & \\
\hline \multicolumn{13}{|c|}{ Satisfacción con la organización } \\
\hline Est oy informado/a sobre los beneficios de esta actividad. & 389 & 1,01 & 3,84 & 1,04 & 3,95 & 0,98 & 0,039 & 4,08 & 0,96 & 3,76 & 1,03 & 0,043 \\
\hline Me gusta el horario en que se desarrolla la actividad. & 4,35 & 0,86 & 4,37 & 0,87 & 4,33 & 0,86 & NS & 4,34 & 0,87 & 4,36 & 0,87 & NS \\
\hline Est oy satisfecho/a con la relación cali dad-precio de la actividad. & 4,09 & 0,89 & 4,03 & 0,97 & 4,15 & 0,8 & NS & 4,11 & 0,91 & 4,07 & 0,88 & NS \\
\hline Existen varios aspectos mejorables en esta actividad. & 3,43 & 1,02 & 3,48 & 1,07 & 3,39 & 0,97 & NS & 3,61 & 0,93 & 3,3 & 1,06 & NS \\
\hline Estoy contento/a con el número de horas semanales dedicadas a esta actividad. & 4,41 & 0,77 & 4,51 & 0,67 & 4,31 & 0,85 & NS & 4,4 & 0,8 & 4,41 & 0,76 & NS \\
\hline \multicolumn{13}{|c|}{ Satisfacción con otros aspectos } \\
\hline Con esta actividad obtengo los resul tados que esperaba. & 4,29 & 0,75 & 4,33 & 0,76 & 4,25 & 0,74 & NS & 4,47 & 0,59 & 4,16 & 0,82 & 0,021 \\
\hline Las sesiones me resultan monótonas. & 2,39 & 1,25 & 2,4 & 1,33 & 2,39 & 1,17 & NS & 2,47 & 1,35 & 2,34 & 1,17 & NS \\
\hline La intensidad de las sesiones es la adecu & 4,17 & 0,79 & 4,16 & 0,83 & 4,17 & 0,74 & NS & 4,27 & 0,83 & 4,09 & 0,75 & NS \\
\hline Me divierto mu cho practican do esta acti vidad. & 4,32 & 0,87 & 4,36 & 0,79 & 4,28 & 0,94 & NS & 4,44 & 0,72 & 4,23 & 0,95 & NS \\
\hline A veces tem ino con dolor de cabeza. & 1,5 & 0,82 & 1,45 & 0,76 & 1,55 & 0,87 & NS & 1,39 & 0,71 & 1,58 & 0,89 & NS \\
\hline \multicolumn{13}{|c|}{ Satisfacción general con la actividad } \\
\hline $\begin{array}{l}\text { A continuación y para terminar, valore su grado de satisfacción general con } \\
\text { esta actividad en la siguiente escala de la sección: }\end{array}$ & 8,11 & 1,24 & 8,22 & 1,04 & 8 & 1,39 & NS & 8,21 & 1,1 & 8,03 & 1,32 & NS \\
\hline
\end{tabular}

de los distintos parámetros evaluados dada la densidad de los resultados derivados. La tabla 1 expone la distribución de muestra en relación al sexo y a la antigüedad/experiencia acumulada en este tipo de práctica. En la tabla 2 se presenta una comparativa de carácter descriptivo de cada uno de los ítems entre los datos de este trabajo y los del el ya mencionado estudio de referencia (Sanz et al., 2005).

La tabla 3 muestra el grado de satisfacción del cliente en relación al monitor, a la sala en la que se practica la actividad y a las bicicletas: su funcionamiento, estado, limpieza y comodidad. La tabla 4 muestra el grado de satisfacción en base a aspectos organizativos de la actividad (información, horario, relación calidad-precio, aspectos a mejorar). En la tabla 4 también se expone el conjunto de ítems que no ha tenido cabida en las anteriores categorías pero que se deben tener en cuenta para valorar el grado de satisfacción del cliente: obtención de los resultados por los que se realiza la actividad, monotonía de las sesiones, la intensidad, diversión y molestias por dolores así como el grado de satisfacción general con la actividad por parte de los usuarios a partir de una escala Likert 0-10. Para los 5 parámetros evaluados a través del cuestionario, se obtuvieron los estadísticos descriptivos así como En todos los casos se ha extraído la media y su desviación estándar el análisis descriptivo se ha llevado a cabo a tres niveles: general, segmentado según tipo de centro y, segmentando según género. Del mismo modo, se aplicó una estadística de contraste para comparar centro grandes vs centros pequeños y hombre vs mujeres para todos los ítems dentro de cada categoría.

Por último, la figura 1 muestra los resultados obtenidos a partir de llevar a cabo el análisis de correlaciones para detectar el nivel de asociación existente entre el grado de satisfacción general del usuario y los distintos ítems que conformaban el cuestionario, así como entre los distintos ítems entre sí; existen correlaciones estadísticamente significativas $(\mathrm{p}<.05)$.

\section{Discusión}

En este estudio se observa que el usuario de esta actividad, en mayor medida de género femenino (58,1\%), es fiel y no esporádico
(53,4 más de un año de práctica). Ellos están más satisfechos que ellas, mientras que el trato ofrecido por el monitor se presenta como una de las acciones más valoradas de cara a la satisfacción del cliente. A nivel general, no se encontraron diferencias importantes entre los denominados centros grandes y centros pequeños ni atendiendo al género. Estudios de este tipo deberían realizarse desde las mismas empresas para conocer la realidad de su empresa así como facilitar la toma de decisiones estratégicas.

El cliente es el eje central de cualquier negocio que ofrece un servicio (Redondo, Olivar y Redondo, 2006; Afthinos, Theodorakis y Nassis, 2005; Alexandris, Dimitriadis y Kasiara, 2001; Granero, Ruiz-Juan, García, Baena y Gómez, 2008). Por eso motivo, además de invertir en campañas de captación de usuarios potenciales, hay que invertir en cuidar y satisfacer al cliente actual. Analizando este tipo resultados se abre un ramillete de posibilidades para optimizar el rendimiento de una empresa de cara al futuro. Esto se plantea como la clave del éxito y supervivencia en una época donde la competencia entre instalaciones es creciente. A este crecimiento de la competencia se le suma la situación de crisis económica general que hace que el consumidor se cuestione mucho más sus gastos. Como dato significativo, y que apoya esta idea, es necesario observar la tabla 1 que expone información acerca de la antigüedad de práctica en la actividad. Cerca de 90 sujetos (60,8\%) acumulan entre nueve meses y más de un año de experiencia practicando ciclismo indoor lo cual demuestra que es una actividad que genera clientes de asistencia duradera y regular, motivo por el que los responsables de los centros deben cuidarla.

En el caso estudiado, ciclismo indoor en Granada, parece que las diferencias más significativas aparecen en relación a la instalación (temperatura, ventilación, aspectos que configuran la comodidad del entorno) y al equipamiento (las bicicletas, limpieza de las mismas, estado y organización). En relación a esteúltimo aspecto cabe destacar la importancia que especialmente las mujeres le dan a la comodidad de las bicicletas. La literatura muestra estudios con resultados parecidos pero no idénticos al presente (Calabuig, Quintanilla y Mundina, 2008). Por otro lado, de forma similar a los que concluyen otros estudios (Dorado y 


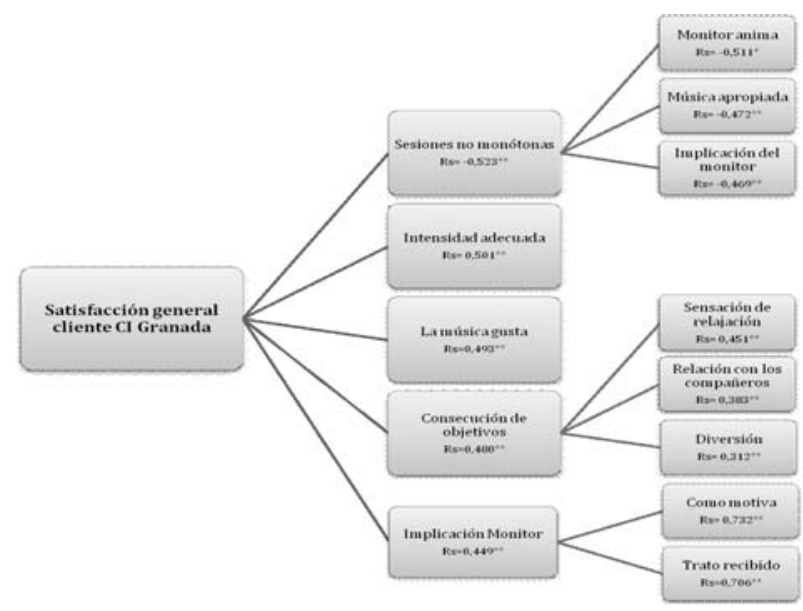

Rs: coefici ente de correlación de Spearmam. *: p<0,05; **: p <0,01; ***: p $<0,001$.

Figura 1. Corelaciones entre satisfacción general del usuario y res to de items $\left({ }^{*} \mathrm{p}<0,05 ; * * * \mathrm{p}<0,01\right)$.

Gallardo, 2004; García y Pires, 2010), el trato ofrecido por el monitor se presenta como una de las acciones más valoradas por el cliente y, por consiguiente, con un importante peso en relación a su grado de satisfacción. La información sobre los beneficios que aporta la actividad también es bien recibida por parte del cliente lo que sugiere que al consumidor le gusta conocer el por qué y el para qué de la actividad que practica.

En relación a los distintos parámetros analizados los resultados reflejan diferencias estadísticamente significativas sólo en 3 de los 27 ítems para los que se aplicó las pruebas de contraste segmentando por tipo de centro (grande vs pequeño). Este dato es susceptible de varias interpretaciones y especulaciones. Por un lado, permite replantear a los gerentes y directores técnicos de las grandes instalaciones que su diferencia de cuota mensual no los distingue en la mayoría de aspectos analizados. De hecho, en ciertos ítems tales como los relacionados con el trato por parte de los monitores ( $\mathrm{p}=.032$ ) y la información sobre los beneficios ( $\mathrm{p}=.039$ ) las diferencias existentes favorecían en este caso a los centros pequeños. La comodidad de la bicicleta fue el único punto que marcó la diferencia a favor de los grandes centros ( $p=.003)$. Sin restar importancia a estos aspectos donde se detectó diferencia estadísticamente significativa, en el resto de ítems existió un equilibrio comparando las instalaciones de centros que cuentan con menos volumen de socios y, como consecuencia, menor potencial económico. Del mismo modo, quedó reflejado que, por un lado, la satisfacción general de los clientes de los grandes centros es ligeramente mayor, lo cual podría ser justificado a partir de las diferencias ya mencionadas. Por tanto, aunque de cara al exterior podría parecer que las diferencias entre grandes y pequeños centros, únicamente por sus posibilidades económicas, debían ser grandes, queda demostrado que para esta muestra concreta no es así.

Por otro lado, estableciendo la comparativa en base al sexo, los hombres están ligeramente más satisfechos que las mujeres con la actividad y sus diferentes variables. Ellos estaban más contentos con el trato del monitor $(\mathrm{p}=.016)$, consideraban las bicicletas más cómodas (.003), se sentían mejor informados ( $\mathrm{p}=.043$ ) y además opinaban de una forma más optimista que ellas acerca de si que cumplían los objetivos previstos para esta actividad ( $\mathrm{p}=.021$ ). En el resto de ítems, volvía a existir un equilibrio entre ellos y ellas. Por último, destacar que son las mujeres, a pesar de ser más exigentes, las que más practican ciclismo indoor en Granada de acuerdo con la muestra analizada.

En cuanto a la comparación de los resultados con los del estudio que usó la misma herramienta por primera vez y que se ha tomado como referencia (Sanz et al., 2005), podemos concluir que no hay ningún aspecto que se haya modificado sustancialmente. Los resultados son muy parecidos a pesar de tratarse de situaciones geográficas y momentos en el tiempo tan diferentes. A partir de esta comparativa nace la duda acerca de si esta situación está motivada por el hecho de que la actividad ha evolucionado poco, o por el contrario, ha ido adaptándose satisfactoriamente a la aparición de nuevas modas, tecnologías y tendencias. En ambos estudios la satisfacción general del cliente con la actividad se encuentra por encima de 8, en el caso de Granada (8.11 \pm 1.24$)$.

Por último, a partir del análisis de correlaciones bivariadas llevado a cabo destacamos que el grado de satisfacción general con la actividad del cliente presentó una correlación positiva y significativa con una serie de aspectos tales como la intensidad de la sesión, la música, el carácter no mónotono de la sesión, el grado de consecución de os objetivos marcados y la implicación del monitor ( $<<.01$, para todos los casos). En relación a esteúltimo aspecto, el trato dispensado al cliente y la forma de motivarlo son determinantes para que el usuario esté satisfecho con su monitor.

Este trabajo no está exento de limitaciones. Una muestra mayor dentro de la misma población podría permitir una mayor representatividad del dato. Del mismo modo, aprovechando la versión online del cuestionario podría haberse difundido la herramienta a través de un link por las bases de datos de las distintas cadenas de centros de fitness que participaron en este estudio piloto, lo cual será tenido en cuenta de cara trabajos posteriores. Por último, consideramos oportuno promover estudios en esta dirección con niños, e incluso plantear la posibilidad la inclusión de este tipo de practica como contenido escolar (Otín, de la Fuente, López, Risco y Gómez, 2013). Como se ha observado, hay una serie de aspectos que el cliente valora en mayor o menor medida y, por tanto, se presentan como claves en la captación y retención de éste, pero no debemos olvidar que para ser detectados primero deben ser considerados objeto de estudio y evaluación.

\section{Conclusiones}

El grado de satisfacción y de fidelización del cliente de ciclismo indoor del área metropolitana de Granada es notable. Se observa que el usuario practicante de esta actividad es fiel y no esporádico. El trato ofrecido por el monitor se presenta como una de las acciones más valoradas en términos de satisfacción del cliente. A nivel general, no se encontraron diferencias importantes entre los denominados centros grandes y centros pequeños. Las mujeres, a pesar de estar menos satisfechas que los hombres, son las que más practican.

\section{Agradecimientos}

Este estudio no se hubiera podido realizar sin la colaboración de los gerentes y directores de los centros de fitness y centros deportivos participantes. Del mismo modo, agradecer a esas 148 personas que han dedicado parte de su tiempo libre a completar el cuestionario. Uno de los autores del presente estudio posee una beca del Ministerio Español deEducación(AP2009-0529).

\section{Referencias}

Afthinos, Y.; Theodorakis, N.D. y Nassis, P. (2005). Customer's expectations of service in Greek fitness centres. Gender, age, type of sport center, and motivation differences. Managing Service Quality, 15, 245-258.

Alexandris, K.; Dimitriadis, N. y Kasiara, A. (2001). The Behavioural Consequences of Perceived Service Quality. An Exploratory Study in the Context of Private Fitness Clubs in Greece. European Sport management Quaterly, 1, 250-281.

Ashby, M. (2000). How to write a paper. Engineering Department, University of Cambridge, Version, 5, 38.

Bodet, G. (2006). Investigating Customer Satisfaction in a Health Club Context. European Sport management Quaterly, 6, 149-165.

Calabuig, F; Quintanilla, I. y Mundina, J. (2008). La calidad percibida de los servicios deportivos: diferencias según instalación, género, edad y tipo de usuario en servicios náuticos. Revista Internacional de Ciencias del Deporte, 4, 25-43.

Dorado, A. y Gallardo, L. (2004). La satisfacción del consumidor deportivo los servicios públicos. Investigación y Marketing, 83, 2630 . 
Granero, A., Ruiz-Juan, F., García, M., Baena, A. y Gómez, M. (2008). Análisis del perfil sociodemográfico de senderistas y ciclistas que recorren el Camino de Santiago. Retos: nuevas tendencias en educación física, deporte y recreación, (13), 56-59.

García Ferrando, M. (2001). Los españoles y el deporte: prácticas y comportamientos en la última década del siglo XX. Encuesta sobre los hábitos deportivos de los españoles, 2000. Ministerio de Educación, Cultura y Deporte. Consejo Superior de Deportes.

García Ferrando, M. (2006). Posmodernidad y Deporte: Entre la individualización y la Masificación. Encuesta hábitos deportivos de los españoles 2005. Madrid, CIS/Siglo XXI

García Ferrando, M., y Llopis Goig, R. (2011). Ideal democrático y bienestar personal: encuesta sobre los hábitos deportivos en España 2010. CIS.

García, J. y Pires, F. (2010). Fidelización de usuarios mayores en centros de fitness: Gestión de clientes por programas de actividad física. Retos. Nuevas tendencias en Educación Física, Deporte y Recreación, 17, 103-106

Lofshult, D. (2003). Indoor cycling is still on a roll. IDEA health y fitness source, 21, 17-21

Luna-Arocas, R. y Mundina, J. (1998). El marketing estratégico del deporte: satisfacción, motivación y expectativas. Revista de Psicología del Deporte (13), 169-174.

Luna, R., Mundina, J. y Gómez, A. (1998). La creación de una escala para medir la calidad de servicio y la satisfacción: el Neptuno-1. Deporte y Calidad de Vida. (279-290). Madrid: Librerías deportivas Esteban Sanz.

Luna, R. y Tang, TL. (2005). The use of cluster analysis to segment clients of a sport centre in Spain. European Sport Management Quarterly. 5, 381-413.

Martínez-Tur, V., Peiró, J. M. y Ramos, J: (2001). Calidad de servicio y satisfacción del cliente. Madrid: Ed Síntesis.

Otero, J. M. (2004). Hábitos y actitudes de los andaluces ante el deporte (2002). Cádiz: Consejería de Turismo y Deporte. Observatorio del Deporte Andaluz.

Otín, C., De la Fuente, F., López, M., Risco, I, y Gómez, S. (2013). Aula en bici: un proyecto longitudinal de intervención docente en Ed. Primaria. Retos. Nuevas tendencias en Educación Física, Deporte y Recreación, (23), 5-9.

Papadimitriou, D, A., y Karteroliotis, K. (2000). The service quality expectations in private sport and fitness centers: A reexamination of the factor structure. Sport Marketing Quarterly, 9, 157-164.

Redondo, J.C., Olivar, D. y Redondo, A. (2006). El Modelo EFQM de gestión de la calidad en las instalaciones deportivas. Sevilla: Wanceulen.

Sanz, I.; Redondo, J. C.; Gutiérrez, P. y Cuadrado, G. (2005). La satisfacción en los practicantes de spinning: Elaboración de una escala para su medición. European Journal of Human Movement, 13, 1736.

Shonk, D. y Chelladurai, P. (2008). Service Quality, Satisfaction, and Intent to Return in Event Sport Tourism. Journal of Sport management, 22 (5), 587-602.

Westerbeek, H. M. y Shilbury, D. (2003). A conceptual model for sport services marketing research: integrating quality, value and satisfaction. International Journal of Sport Marketing y Sponsorship, March-April, 11-31.

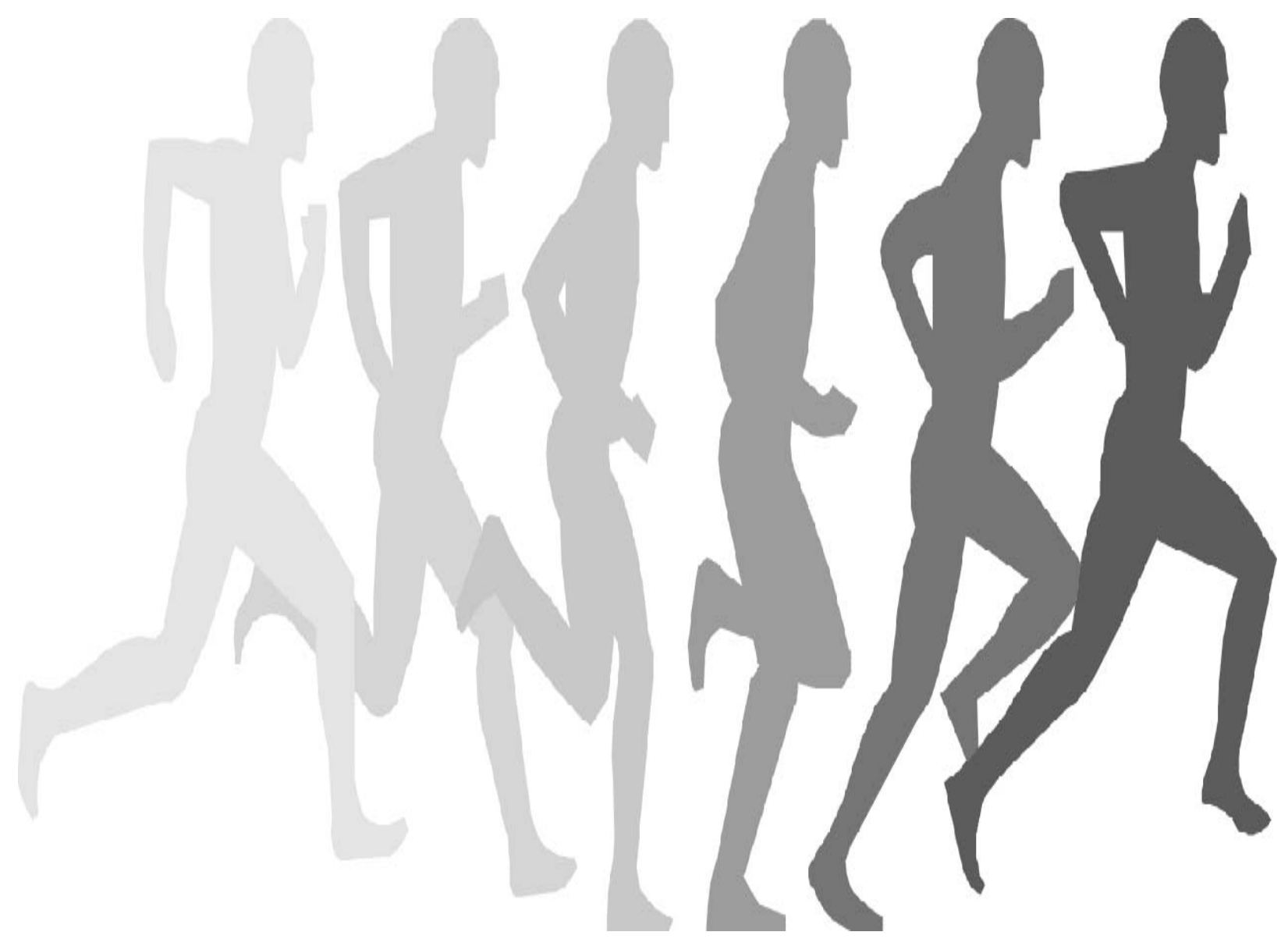

